Review

\title{
Liposarcoma: Advances in Cellular and Molecular Genetics Alterations and Corresponding Clinical Treatment
}

\author{
Lingge Yang ${ }^{1,2}$, Shiqi Chen ${ }^{1,2}$, Peng Luo ${ }^{1,2}$, Wangjun Yan ${ }^{1}{ }^{\bowtie}$, Chunmeng Wang ${ }^{1,2}{ }^{\bowtie}$ \\ 1. Department of Musculoskeletal Oncology, Fudan University Shanghai Cancer Center, Shanghai, China \\ 2. Department of Oncology, Shanghai Medical College, Fudan University, Shanghai, China
}

$\square$ Corresponding authors: Dr. Chun-meng Wang, MD \& Wangjun Yan, MD, Department of Musculoskeletal Oncology, Fudan University Shanghai Cancer Center; Department of Oncology, Shanghai Medical College, Fudan University, Shanghai 200032, China. Fax: 86-21-64430130. E-mail: cmwang1975@163.com \& yanwj@fudan.edu.cn.

(0) The author(s). This is an open access article distributed under the terms of the Creative Commons Attribution License (https://creativecommons.org/licenses/by/4.0/). See http://ivyspring.com/terms for full terms and conditions.

Received: 2019.05.06; Accepted: 2019.09.12; Published: 2020.01.01

\begin{abstract}
Liposarcoma is a malignant tumor of mesenchymal origin with significant tissue diversity. It is composed of adipocytes with different degrees of differentiation and different degrees of heteromorphosis. It is not sensitive to traditional radiotherapy and chemotherapy and has a poor prognosis. In recent years, with the rapid development of basic immunology, molecular genetics and tumor molecular biology, the histological classification of liposarcoma has become increasingly clear. More and more new methods and technologies, such as gene expression profile analysis, the whole genome sequencing, miRNA expression profile analysis and RNA sequencing, have been successfully applied to liposarcoma, bringing about a deeper understanding of gene expression changes and molecular pathogenic mechanisms in the occurrence and development of liposarcoma. This study reviews the present research status and progress of cellular and molecular alterations of liposarcoma and corresponding clinical treatment progress.
\end{abstract}

Key words: Liposarcoma; Cytogenetics; Molecular genetics; Epigenetics; Clinical Treatment

\section{Introduction}

Liposarcoma is a common type of soft tissue sarcoma that accounts for about $20 \%$ [1] of all adult sarcomas. Liposarcoma often develops in deep soft tissues of lower limbs and retroperitoneal parts, accounting for $24 \%$ and $45 \%$ [2] of limb sarcomas and retroperitoneal soft tissue sarcomas respectively. In accordance with the typical morphological characteristics and biochemical characteristics showed at different stages of adipocyte differentiation, liposarcoma is divided into 3 groups and 5 types by WHO: well-differentiated/ dedifferentiated liposarcoma (WDL/DDL), myxoid/round-cell liposarcoma (MRCL) and pleomorphic liposarcoma (PLS) [3]. Such kind of classification tries to determine different subtypes of liposarcoma according to their clinical features, morphological features, immunophenotypic and genetic characteristics, etc., making liposarcoma become an independent disease species to facilitate research and comparison of clinical, pathological, genetic data and molecular pathogenesis. This study reviews the cellular and molecular genetics alterations and corresponding clinical treatment of liposarcoma.

\section{Well-differentiated/Dedifferentiated Liposarcoma (WDL/DDL)}

WDL/DDL is most common type of liposarcoma, accounting for about $40 \%$ to $45 \%$ [4] of liposarcoma. Superficial WDL is also called atypical lipomatous tumor (ALT). Histologically, WDL consists mainly of mature adipocytes, atypical stromal cells, and a small number of scattered fat mother cells, 
which is quite similar to normal adipose tissue and mature benign lipoma tissues. Cytogenetic studies have showed that WDL/DDL is characterized by supernumerary ring chromosome and/or giant marker chromosome composed of amplified products from the q13-15 region on Chromosome No. 12 [5]. Many genes, such as MDM2, CDK4, HMGA2, CPM, SAS/TSPAN31, DYRK2, YEATS4 and others, amplify along with WDL/DDL [6].

Studies have showed that gene amplification exists in the 12q12-21 and 10p11-14 regions of the WDL/DDL cell lines [7]. Among them, MDM2 and CDK4 keep being amplified and expressed. Both genes are proto-oncogenes, and the encoded proteins are involved in the regulation of cell cycles. MDM2 expression products are also transcriptional activation inhibitors of p53, which inhibit the transcription of p53 and lead to cell proliferation [4, 5, 7-11]. HMGA2 expression products can regulate transcription through DNA structural modification and cross-linking with other enhanceosome proteins, which are normally expressed during embryonic development instead of normal somatic cells. However, for WDL/DDL, MDM2 amplification is accompanied by HMGA2 dysregulation, presenting its oncogenic property [8]. CPM is at the downstream location of MDM2 and the encoded protein is associated with many functions, such as adipose tissue differentiation, osteogenic differentiation, inflammation, and coagulation [9]. FRS2 expression products can be activated into fibroblast growth factor receptor (FGFR) signal, and the abnormal activation of this signal can lead to tumor formation, tumor angiogenesis, and metastasis [10,11]. A study found that FRS2 was amplified in 93.2\% (132/146) of WDL/DDL, and the FRS2/CEP12 ratio in DDL was significantly higher than that in WDL $(\mathrm{P}=0.0005)$ [12].

WDL is a well-differentiated type of liposarcoma with comparatively weak invasive ability basically without metastasis. However, WDL can be dedifferentiated and be converted to DDL, so as to obtain stronger invasive ability with potential local recurrence and distant metastasis. This dedifferentiation occurs in approximately $10 \%$ of WDL [13]. DDL morphologically consists of WDL region and a suddenly-transitioned region of non-adipose tissue sarcoma. Thus, it is not difficult to understand that DDL has the same cellular and molecular genetic characteristics as WDL.

The difference is that DDL has additional genetic changes, especially co-amplification of genes in chromosome $6 \mathrm{q} 23$ and $1 \mathrm{p} 32$ regions, such as JUN and ASK1/MAP3K5 [5, 7, 13-16]. Both of the two gene-encoded products can participate in the conduction of c-Jun N-terminal protein kinase (JNK) signal pathway. JUN expression products can regulate the activity of factors involved in adipocyte transcription, and ASK1/MAP3K5 can encode the kinase in the upstream location of JUN. The amplification of JUN or ASK1/MAP3K5 is related to the fact that WDL dedifferentiation leads to the tissue type changing to DDL [15]. However, in a phase II clinical trial, plitidepsin, which activated the JUN pathway to induce apoptosis, did not show ideal clinical effect in treating patients with advanced DDL [16]. The systematic connection of the amplification of both genes and DDL occurrence has not been fully confirmed.

In addition, DDIT3, PTPRQ, YAP1, and C/EBPa also have different degrees of amplification. The copy number and mRNA levels of these four genes are correlated with the expression level of JUN [17]. The transcription factor, C/EBPa, which is involved in cell cycle regulation and cell differentiation, is studied more frequently. Its lack of expression is an important factor for DDL to maintain differentiation and inhibit apoptosis [18]. Besides, the expression of anti-aging protein Klotho down-regulates in DDL compared with WDL and adipose tissue, which is related to poor prognosis; in addition, it can regulate the drug sensitivity of thapsigargin and gemcitabine by inhibiting ERK1/2 signal transduction, which provides a new therapeutic strategy for DDL [19].

\section{Myxoid and Round-Cell Liposarcoma (MRCL)}

MRCL is the second largest category of liposarcoma. It is usually composed of round cell liposarcoma (RCL) and myxoid liposarcoma (MLS) in histomorphology. In general, RCL is more invasive than MLS and the higher the proportion of the former is, the worse the prognosis is indicated [20]. The most prominent cytogenetic feature of MRCL is that about $95 \%$ [21] of cases have specific $t(12 ; 16)(q 13 ; p 11)$ chromosomal translocation, which produces FUS-DDIT3 fusion protein (also known as TLS-CHOP fusion protein), while about $5 \%$ [22] of cases have $\mathrm{t}(12 ; 22) \quad(\mathrm{q} 13 ; \mathrm{q} 12)$ chromosomal translocation, producing EWSR1-DDIT3 fusion protein.

FUS-DDIT3 and EWSR1-DDIT3 show a high degree of specificity and can be used as characteristic diagnostic indicator. These fusion proteins are important molecules for development of sarcoma and inhibition of adipogenesis and play crucial roles in the pathogenesis of MRCL. The expression level of FUS-DDIT3 fusion protein is also positively correlated with cell differentiation [23]. In addition, studies have shown that the FUS-DDIT3 fusion gene can enhance the invasion ability of MRCL by activating the SRC/FAK/RHO/ROCK signal axis, and the 
expression level of FAK is related to the degree of malignancy and the tumor grade [24].

Interestingly, for most of MRCL, TP53 is not mutated and can produce functionally normal p53, and once this gene is mutated, the invasive ability of MRCL will also be strengthened [25].

It has been shown that MRCL can cause gene mutation of EGFR, PDGFRB, RET, MET and VEGFR1 through the interaction of the autocrine/paracrine loop and the receptor tyrosine kinase (RTK), and it can keep activating the signaling pathway of the downstream PI3K/Akt, leading to the over-expression of growth factor receptor RET and IGF1R, which is related to the transformation of MLS to $\mathrm{RCL}$, increasing invasiveness, and poor prognosis [20, 26-28].

Round cell took up more than 5\% of MRCL, indicating a poor prognosis [29]. In order to further investigate the process of MLS transforming into RCL, Cecco et al. [30] used gene expression profiling, immunohistochemistry, biochemical analysis, and other techniques to study two groups of samples containing only MLS and RCL, respectively, and found that in this process, the silence of the DLK1-DIO3 genomic region at $14 \mathrm{q} 32$ resulted in the over-expression of genes such as YY1/C-MYC/HDAC2 that promoted rapid cell cycle progression. And MKNK2, MSX1 and TRIM71 encoded for cell proliferation and stem cell formation, were also over-expressed. MLS developed into RCL by crossing epigenetic silencing restriction point, rearranging its stem cell sample differentiation markers.

Nezu et al. [31] used miRNA microarray analysis to explore the transformation process and found that miR-135b was expressed at a higher level in RCL, which could be used as an oncogenic miRNA. Through the miR-135b/THBS2/MMP2 axis, miR-135b strengthened the ability of MLS to grow, invade and metastasize. At the same time, the density of MLS cells increased and the extracellular collagen matrix decreased, resulting in a change in the histopathology of MLS and eventually transformation to RCL.

\section{Polymorphic Liposarcoma (PLS)}

PLS accounts for less than 5\% [32] of liposarcoma, which is the rarest type. With high local recurrence rate and distant metastasis rate, PLS is more invasive than other types of liposarcoma, and is less sensitive to conventional treatment. Histologically, PLS is composed of many irregular cell groups and abundant isolated, non-adherent cells and has distinct polymorphism and common characteristic sheets of bizarre pleomorphic mono or multivacuolated adipoblasts [33-35].
The cytogenetic feature of PLS is complex aneuploid karyotypes with complex genomic amplification and deletions [34, 36]. Barretina et al. [37] found mutations in genes such as TP53, RB1 and NF1 in PLS by DNA sequencing, while Ghadimi et al. [36] found many biomarkers, such as peroxisome proliferator-activated receptor gamma (PPAR- $\gamma$ ), VEGF, survivin protein, B-cell leukemia 2 and matrix metalloproteinase 2, were over-expressed in PLS; in addition, they observed the presence of high-frequency deletion of retinoblastoma protein and high-frequency gene mutation of TP53 (about $60 \%)$ in PLS.

In general, although many chromosome structures and gene expression abnormalities have been discovered in PLS, no characteristic or constant chromosomal aberrations or molecular alterations have been found.

\section{The Research Significance}

\section{Diagnosis and Differential Diagnosis}

In WDL/DDL, continuous amplification of CPM can be detected in all tissues and can be used as a new indicator for diagnosis [9]. The cancer testis antigen NY-ESO-1 seems to be a useful immunohistochemical marker to support the diagnosis of MRCL because of its high sensitivity and specificity [38].

In terms of differential diagnosis, it is difficult to diagnose only from the histomorphological manifestations of WDL and DDL. The current diagnostic method is guided by MDM2 and CDK4 immunohistochemistry and determined by the amplification of the corresponding genes. Recently, p16 immunohistochemistry has been considered to be an effective diagnostic biomarker. A study has shown that $68 \%$ of WDL and $72 \%$ of DDL could express the above three proteins, while all WDL and $93 \%$ of DDL expressed at least two of them. Through performing MDM2 immunohistochemical test on both, they found that the sensitivity and specificity were $86 \%$ and $74 \%$, while CDK4 was detected to be $86 \%$ and $89 \%$, p16 was detected to be $93 \%$ and $92 \%$, and the the sensitivity and specificity was $71 \%$ and $98 \%$ when they combined detection of the three markers [39]. In conclusion, the study suggested that MDM2, CDK4, and p16 immunohistochemical detection were effective supplementary means of identifying WDL and DDL from other types of liposarcoma.

P16 is gene-encoded by CDKN2A and inhibits the progression of the cell cycle by binding to CDK4, which is the most sensitive and specific marker for detecting WDL/DDL. However, Kang et al. [40] believed that when distinguishing retroperitoneal DDL from other common retroperitoneal tumors, 
especially leiomyosarcoma and desmoid tumors, p16 is not as practical as MDM2 and CDK4 due to its low specificity.

Some researchers further analyzed the three proteins above and found that MDM2+/p16+ were all WDL, MDM2-/p16- was benign fatty tumor, MDM2/CDK4/p16 was DDL, and MDM2-/CDK4/p16- was a kind of undifferentiated sarcoma. The results showed that the combination of three proteins test could effectively identify WDL and DDL [41].

\section{To Find Suitable Therapeutic Targets}

Clinically, the curative management for localized disease is surgical resection, combined with or without radiotherapy. Systemic treatment with chemotherapy and molecular targeted agents is one of the main therapeutic modalities in patients with advanced or metastatic disease. With increasing number of studies in molecular basis of pathogenesis and emerging new therapeutic targets, the treatment outcome of liposarcoma will be greatly improved in the future.

In WDL/DDL, CDK4 is continuously amplified in about $90 \%$ of cases, and a CDK4/6 inhibitor, palbociclib, showed certain efficacy in the treatment of advanced CDK4+ WDL/DDL, for the $66 \%$ progression-free survival (PFS) rate at 12 weeks, and the median PFS was 18 weeks [42]. Subsequent researches have also been carried out gradually. Some researchers found that palbociclib combined with recombinant methioninase had excellent anti-tumor activity in the doxorubicin-resistant patient-derived orthotopic xenograft animal model [43]. A study reported that the therapeutic effect of CDK4/6 inhibitors on MDL/DDL needed to be achieved by down-regulating the expression of MDM2 protein by PDLIM7 and CDH18 [44].

The clinical study of MDM2 as a therapeutic target for WDL/DDL was reported in 2012. Ray-Coquard et al. [45] used MDM2 antagonist (RG7112) to treat 20 patients, of which one patient got complete response (CR) and 14 patients got stable disease (SD), showing a certain clinical efficacy. However, the adverse reactions were serious, which limited its clinical application. Subsequently, MDM2 inhibitors were also used in clinical trials. A phase I clinical trial of ALRN-6924 in the treatment of advanced solid tumors has achieved some outcomes according to its preliminary report, of which one patient with liposarcoma received partially responsive (PR) [46]. Similarly, one patient with DDL received PR in a phase I clinical trial of DS-3032b for WDL/DDL [47]. According to the results, compared with RG7112, the latter two had fewer adverse reactions and were more suitable for clinical use.
Further detailed reports and clinical trials are worth looking forward to.

It is worth mentioning that although both MDM2 and CDK4 have corresponding targeted inhibitors for clinical trials, in vitro experiments have shown that the cytotoxicity of the two drugs against sarcoma cell lines is mutually antagonistic [48]. Therefore, careful consideration must be given to the combination of CDK4 and MDM2 inhibitors to treat WDL/DDL.

In DDL, many other potential therapeutic targets have been reported. FRS2 is often amplified, and its encoded protein is not normally present in normal fat or preadipocytes, so FRS2 may be an effective target for this type of liposarcoma [10, 11]. In vitro and in vivo experiments have shown that pan-FGFR inhibitor LY2874455 has clinical value in the treatment of DDL for FRS2 amplification [49]. In addition, methylation of $\mathrm{C} / \mathrm{EBPa}$ is found in $24 \%$ of DDL while demethylation pharmacotherapy can restore the expression of C/EBPa in DDL cells so as to inhibit the proliferation of DDL cells in vitro experiment as well as promote apoptosis and slow down the tumor growth in vivo [50]. It suggests that demethylating agent may be a potential therapeutic agent for DDL. Some researchers also found that STAT6 was located in $12 q 13$ and was amplified in about $11 \%$ of DDL [51]. The encoded product was STAT6, a member of the STAT family, which was cytoplasmic transcription factor. The over-expression of this transcription factor was closely related to tumor growth and its inhibitor was of potential therapeutic value to this type of DDL.

Immune checkpoint inhibitors have opened up a new way for the treatment of DDL. Studies have shown that PD-L1 is highly expressed in DDL cell lines, and patients with the PD-L1 expression $\geq 1 \%$ have a significant decrease in recurrence-free survival $(P=0.027)$ and overall survival $(P=0.017)$. They also found that PD-L1 expression is mediated by IFN- $\gamma$, suggesting the possibility of combined treatment for DDL [52]. A phase II clinical trial of pembrolizumab in the treatment of advanced sarcoma has been reported. Of ten patients with DDL enrolled, two cases received PR, four received stable disease (SD), median PFS was 25 weeks, and PFS rate was $60 \%$ at 12 weeks, which has clinical application value [53].

In MRCL, Pollack et al. [54] used immunohistochemistry and qPCR to detect 25 samples and found NY-ESO-1 expression in all their samples. They also found that the sensitivity of MRCL cell lines to antigen-specific lysis was demonstrated by using NY-ESO-1 specific, CD8+ T-cells, suggesting that this antigen should be used as a potential therapeutic target for MRCL. Subsequently, they reviewed the efficacy of CMB305, a therapeutic 
vaccine targeting NY-ESO-1, indicating that the vaccine can significantly improve the OS of MRCL patients [55]. Hemminger et al. [56] also got similar conclusions and they found that CTAG1B and its mRNA were highly over-expressed in MRCL. These proteins also have potential value for targeted immunotherapy.

In addition to NY-ESO-1, other biomarkers also can be used as therapeutic targets for MRCL. Experiments showed that IGF-IR inhibitors could suppress the growth of MRCL cell lines, and IGF-IR/PI3K/Akt signaling pathway could be used as a specific therapeutic target of MRCL [26]. HSP90 inhibitor could block the phosphorylation of ERBB3 and RET in MRCL, leading to tumor tissue necrosis, which was confirmed in vitro and in vivo experiments, thus HSP90 inhibitor was expected to become a medicine for treating MRCL [27]. In MRCL tissue, CD68+ macrophage infiltration indicated a poor prognosis, and the possible mechanism could be that the secretion of heparin-binding EGF-like growth factor (HB-EGF) combines EGFR, resulting in a stronger invasive ability of MRCL invasion [28]. Therefore, HB-EGF and EGFR also have the potential to be therapeutic targets of MRCL.

It has been reported in a case that using apatinib, a small molecule inhibitor of receptor protein tyrosine kinase, targeting VEGFR-1, VEGFR-2, PDGFRB, etc., for the treatment of advanced RCL, the patient achieved PR [57]. There was also a case report showed that a patient with advanced PLS who had failed multiple chemotherapy regimens previously, achieved 3-month PFS and a high quality of life after received apatinib. Apatinib ehxibits a certain clinical efficacy in the treatment of liposarcoma [58].

The ongoing and upcoming clinical trials of targeted therapy and immunotherapy for liposarcoma are detailed in Table 1.

Table 1. Ongoing and Upcoming Clinical Trials of Targeted Therapy and Immunotherapy for Liposarcoma

\begin{tabular}{|c|c|c|c|c|c|}
\hline Drug Name/Code & Targets & Pathological subtypes of liposarcoma & Recruitment & Phase & $\begin{array}{l}\text { ClinicalTrials. } \\
\text { gov ID }\end{array}$ \\
\hline APX005M & CD40 & Well/Dedifferentiated liposarcoma & Not yet recruiting & II & NCT03719430 \\
\hline Ribociclib/LEE011 & CDK4/6 & All & Recruiting & $\mathrm{Ib}$ & NCT03009201 \\
\hline Abemaciclib & CDK4/6 & Dedifferentiated liposarcoma & Recruiting & II & NCT02846987 \\
\hline Ribociclib/LEE011 & CDK4/6 & Well/Dedifferentiated liposarcoma & Recruiting & II & NCT03096912 \\
\hline Ribociclib/LEE011+Everolimus & $\mathrm{CDK} 4 / 6+\mathrm{mTOR}$ & Dedifferentiated liposarcoma & Recruiting & II & NCT03114527 \\
\hline Regorafenib & c-Kit, B-Raf, Raf-1, RET, VEGFR1-3, PDGFR $\beta$ etc. & All & Recruiting & II & NCT02048371 \\
\hline Sitravatinib/MGCD516 & c-Kit, PDGFR a- $\beta$, c-Met, Axl etc. & Well/Dedifferentiated liposarcoma & Recruiting & II & NCT02978859 \\
\hline Selinexor/KPT-330 & CRM1 & Dedifferentiated liposarcoma & Recruiting & II/III & NCT02606461 \\
\hline Selinexor/KPT-330+Ixazomib & CRM1+20S proteasome & Dedifferentiated liposarcoma & Not yet recruiting & I & NCT03880123 \\
\hline Itacitinib/INCB39110 & Jak1 & Myxoid/round cell liposarcoma & Not yet recruiting & I & NCT03670069 \\
\hline MAGE-A44 ${ }^{\mathrm{c}^{1032} \mathrm{~T}}$ cells & MAGE-A4 & Myxoid/round cell liposarcoma & Recruiting & I & NCT03132922 \\
\hline HDM201+Ribociclib/LEE011 & MDM2+CDK4/6 & Well/Dedifferentiated liposarcoma & $\begin{array}{l}\text { Active, not } \\
\text { recruiting }\end{array}$ & $\mathrm{Ib} / \mathrm{II}$ & NCT02343172 \\
\hline $\begin{array}{l}\text { CD8+ NY-ESO-1-Specific T } \\
\text { Cells+LV305 } \pm \text { CMB305 }\end{array}$ & NY-ESO-1 & Myxoid liposarcoma & Recruiting & I & NCT03450122 \\
\hline NYCE T Cells & NY-ESO-1 & Myxoid/round cell liposarcoma & Recruiting & I & NCT03399448 \\
\hline CMB305 \pm Atezolizumab & NY-ESO-1士PD-L1 & Myxoid/round cell liposarcoma & $\begin{array}{l}\text { Active, not } \\
\text { recruiting }\end{array}$ & II & NCT02609984 \\
\hline NY-ESO-1 ${ }^{\mathrm{c} 259} \mathrm{~T}$ cells & NY-ESO-1 & Myxoid/round cell liposarcoma & Recruiting & II & NCT02992743 \\
\hline Pembrolizumab & PD-1 & All & Not yet recruiting & II & NCT03899805 \\
\hline Pembrolizumab & PD-1 & Myxoid/round cell liposarcoma & Recruiting & II & NCT03063632 \\
\hline Nivolumab+Nab-rapamycin & PD-1+mTOR & All & Recruiting & $\mathrm{Ib}$ & NCT03190174 \\
\hline Nivolumab \pm Ipilimumab & PD- $1 \pm$ CTLA- 4 & $\begin{array}{l}\text { Dedifferentiated liposarcoma of the } \\
\text { retroperitoneum }\end{array}$ & Recruiting & II & NCT03307616 \\
\hline Olaratumab & PDGFR a & All & $\begin{array}{l}\text { Active, not } \\
\text { recruiting }\end{array}$ & III & NCT02451943 \\
\hline Olaratumab & PDGFR a & $\begin{array}{l}\text { Myxoid/round cell, pleomorphic or } \\
\text { dedifferentiated liposarcoma }\end{array}$ & Recruiting & II & NCT02584309 \\
\hline Efatutazone & PPAR- $\gamma$ & Myxoid liposarcoma & $\begin{array}{l}\text { Active, not } \\
\text { recruiting }\end{array}$ & II & NCT02249949 \\
\hline Pazopanib & VEGFR 1-3, c-Kit \& PDGF-R & All & Recruiting & II & NCT01532687 \\
\hline Pazopanib & VEGFR 1-3, c-Kit \& PDGF-R & Dedifferentiated, or myxoid liposarcoma & Recruiting & II & NCT02357810 \\
\hline Lenvatinib & VEGFR $2 / 3$ & $\begin{array}{l}\text { Dedifferentiated, myxoid, or } \\
\text { pleomorphic liposarcoma }\end{array}$ & Recruiting & $\mathrm{Ib} / \mathrm{II}$ & NCT03526679 \\
\hline
\end{tabular}




\section{Efficacy Assessment and Prognosis Prediction}

In recent years, with the extensive application of RNA library depth sequencing technology and DNA microarray technology, miRNA has become a hot spot for studying the progress and prognosis of liposarcoma. The core role of miR-143 in the occurrence and development of WDL/DDL has been confirmed [59]. Ugras et al. [60] found that miR-143 was highly expressed in normal adipocytes, while the expression was down-regulated in WDL, and if the expression was further down-regulated, the WDL could develop to DDL; in addition, recovery of miR-143 expression in DDL could inhibit its proliferation and induce apoptosis. Similarly, over-expression of miRNA-133a could inhibit the proliferation and regulate mitochondrial function and glycolysis ability of DDL cell lines. However, in vivo experiments showed that exogenous recombinant miRNA-133a had only the ability to regulate cell metabolism, and without proliferation inhibition [61]. Recently, Mazzu et al. [62] has found that miR-193b regulates multiple oncogenic signaling pathways (such as PDGFR, TGF, and Wnt) by targeting PDGFR- $\beta$, SMAD4 and YAP1 proteins in vitro, and thus miR-193b plays a tumor-suppressing role in the WDL/DDL cell lines.

Borjigin et al. [63] found that the expression of miR-486 could be inhibited by the specific fusion protein FUS-DDIT3 in MRCL, while the addition of exogenous miR-486 could inhibit the growth of MRCL cells. Similarly, miR-145 and miR-451, members of miRNA with tumor-suppressing function, could inhibit the proliferation and differentiation of all types of liposarcoma and induce apoptosis by their over-expression in vitro [64]. The aforementioned miRNA with tumor-suppressing function are good indicators of efficacy evaluation and prognosis, as well as potential therapeutic targets.

Zhang et al. [65] first reported that miR-155 had the important function of carcinogenesis in WDL/DDL, and miR-155 could strengthen the conduction of $\beta$-catenin signaling pathway through direct control of casein kinase 1a (CK1a), and increased the expression of cyclin D1, thus leading to the proliferation of DDL cell lines and accelerating cell cycle progression. Therefore, miR-155 could be a predictor of efficacy and prognosis of WDL/DDL, which was also further confirmed in other researchers' studies [66, 67].

Lee et al. [68] found the over-expression of miR-26a-2 was significantly associated with poor prognosis of patients with WDL/DDL and MRCL $(\mathrm{P}<0.05$ in WDL/DDL group, $\mathrm{P}<0.001$ in $\mathrm{MRCL}$ group). Exosomes-derived miR-25-3p and miR-92a-3p were found in liposarcoma, which could accelerate the proliferation, invasion, and metastasis of liposarcoma by stimulating the secretion of pro-inflammatory cytokine IL-6; in addition, these miRNAs could effectively distinguish patients with liposarcoma from healthy individuals and had the possibility of becoming a new non-invasive biomarker, so as to be used for early diagnosis of liposarcoma, evaluation of efficacy and prognosis [69].

\section{Conclusions}

In the past decade, an in-depth research on the cellular and molecular pathogenesis of liposarcoma has brought about new ideas and methods for clinical diagnosis, treatment and prognosis. Although we are still at the early stage of translating these studies of cellular and molecular level into clinical applications, more and more new methods and technologies have been proposed and applied, which brings hope to patients and medical personnels. The advent of the era of precision medicine calls for further exploration of the pathogenesis of liposarcoma from cellular and molecular level so as to provide patients with individualized and accurate treatment.

\section{Competing Interests}

The authors have declared that no competing interest exists.

\section{References}

1. Codenotti S, Vezzoli M, Monti E, et al. Focus on the role of Caveolin and Cavin protein families in liposarcoma. Differentiation. 2017; 94: 21-6.

2. Huang X, Xiao F, Wang $S$, et al. G protein pathway suppressor 2 (GPS2) acts as a tumor suppressor in liposarcoma. Tumor Biol. 2016; 37(10): 13333-43.

3. Jo VY, Fletcher CD. WHO classification of soft tissue tumours: an update based on the 2013 (4th) edition. Pathology. 2014; 46(2): 95-104.

4. Briski LM, Jorns JM. Primary breast atypical lipomatous tumor/well-differentiated liposarcoma and dedifferentiated liposarcoma. Arch Pathol Lab Med. 2018; 142(2): 268-74.

5. Nishio J, Aoki M, Nabeshima K, et al. Cytogenetic and molecular cytogenetic findings in giant dedifferentiated liposarcoma of the thigh. Oncol Rep. 2011; 27(3): 764-8.

6. Guan $\mathrm{Z}, \mathrm{Yu} \mathrm{X}, \mathrm{Wang} \mathrm{H}$, et al. Advances in the targeted therapy of liposarcoma. Oncotargets Ther. 2015; 8: 125-36.

7. Pedeutour F, Maire G, Pierron A, et al. A newly characterized human well-differentiated liposarcoma cell line contains amplifications of the 12q12-21 and 10p11-14 regions. Virchows Arch. 2012; 461(1): 67-78.

8. Italiano A, Bianchini L, Keslair F, et al. HMGA2 is the partner of MDM2 in well-differentiated and dedifferentiated liposarcomas whereas CDK4 belongs to a distinct inconsistent amplicon. Int J Cancer. 2008; 122(10): 2233-41.

9. Erickson-Johnson MR, Seys AR, Roth CW, et al. Carboxypeptidase M: a biomarker for the discrimination of well-differentiated liposarcoma from lipoma. Mod Pathol. 2009; 22(12): 1541-7.

10. Zhang $\mathrm{K}, \mathrm{Chu} \mathrm{K}, \mathrm{Wu} \mathrm{X}$, et al. Amplification of FRS2 and Activation of FGFR/FRS2 Signaling Pathway in High-Grade Liposarcoma. Cancer Res. 2013; 73(4): 1298-307.

11. Wang X, Asmann YW, Erickson-Johnson MR, et al. High-resolution genomic mapping reveals consistent amplification of the fibroblast growth factor receptor substrate 2 gene in well-differentiated and dedifferentiated liposarcoma. Genes Chromosomes Cancer. 2011; 50(11): 849-58. 
12. Jing W, Lan $\mathrm{T}$, Chen $\mathrm{H}$, et al. Amplification of FRS2 in atypical lipomatous tumour/well-differentiated liposarcoma and de-differentiated liposarcoma: a clinicopathological and genetic study of 146 cases. Histopathology. 2018; 72(7): 1145-55.

13. Thway K, Jones RL, Noujaim J, et al. Dedifferentiated liposarcoma: updates on morphology, genetics, and therapeutic strategies. Adv Anat Pathol. 2016; 23(1): 30-40.

14. Tap WD, Eilber FC, Ginther C, et al. Evaluation of well-differentiated/de-differentiated liposarcomas by high-resolution oligonucleotide array-based comparative genomic hybridization. Genes Chromosomes Cancer. 2011; 50(2): 95-112.

15. Saâda-Bouzid E, Burel-Vandenbos F, Ranchère-Vince D, et al. Prognostic value of HMGA2, CDK4, and JUN amplification in well-differentiated and dedifferentiated liposarcomas. Mod Pathol. 2015; 28(11):1404-14.

16. Toulmonde M, Le Cesne A, Piperno-Neumann S, et al. Aplidin in patients with advanced dedifferentiated liposarcomas: a French Sarcoma Group Single-Arm Phase II study. Ann Oncol. 2015; 26(7): 1465-70.

17. Abeshouse A, Adebamowo C, Adebamowo SN, et al. Comprehensive and integrated genomic characterization of adult soft tissue sarcomas. Cell. 2017; 171(4): 950-65.

18. Wu YV, Okada $\mathrm{T}$, DeCarolis $\mathrm{P}$, et al. Restoration of $\mathrm{C} / \mathrm{EBPa}$ in dedifferentiated liposarcoma induces G2/M cell cycle arrest and apoptosis. Genes Chromosomes Cancer. 2012; 51(4): 313-27.

19. Delcroix V, Mauduit $\mathrm{O}$, Tessier $\mathrm{N}$, et al. The role of the anti-aging protein Klotho in IGF-1 signaling and reticular calcium leak: impact on the chemosensitivity of dedifferentiated liposarcomas. Cancers. 2018; 10(11): 439.

20. Qi $\mathrm{Y}, \mathrm{Hu} \mathrm{Y}$, Yang $\mathrm{H}$, et al. Establishing a patient-derived xenograft model of human myxoid and round-cell liposarcoma. Oncotarget. 2017; 8(33): 54320-30.

21. Kanojia D, Nagata Y, Garg M, et al. Genomic landscape of liposarcoma. Oncotarget. 2015; 6(40): 42429-44.

22. Iwasaki H, Ishiguro M, Nishio J, et al. Extensive lipoma-like changes of myxoid liposarcoma: morphologic, immunohistochemical, and molecular cytogenetic analyses. Virchows Arch. 2015; 466(4): 453-64.

23. Åman P, Dolatabadi S, Svec D, et al. Regulatory mechanisms, expression levels and proliferation effects of the FUS-DDIT3 fusion oncogene in liposarcoma. J Pathol. 2016; 238(5): 689-99.

24. Tornin J, Hermida-Prado F, Padda RS, et al. FUS-CHOP promotes invasion in myxoid liposarcoma through a SRC/FAK/RHO/ROCK-dependent pathway. Neoplasia. 2018; 20(1): 44-56.

25. Ståhlberg A, Kåbjörn Gustafsson C, Engtröm K, et al. Normal and functional TP53 in genetically stable myxoid/round cell liposarcoma. PLoS One. 2014; 9(11): e113110.

26. Trautmann $M$, Menzel J, Bertling $C$, et al. FUS-DDIT3 fusion protein-driven IGF-IR signaling is a therapeutic target in myxoid liposarcoma. Clin Cancer Res. 2017; 23(20): 6227-38.

27. Safavi S, Järnum S, Vannas C, et al. HSP90 inhibition blocks ERBB3 and RET phosphorylation in myxoid/round cell liposarcoma and causes massive cell death in vitro and in vivo. Oncotarget. 2016; 7(1): 433-45.

28. Nabeshima A, Matsumoto Y, Fukushi J, et al. Tumour-associated macrophages correlate with poor prognosis in myxoid liposarcoma and promote cell motility and invasion via the HB-EGF-EGFR-PI3K/Akt pathways. Br J Cancer. 2015; 112(3): 547-55.

29. Moreau L, Turcotte R, Ferguson P, et al. Myxoid \round cell liposarcoma (MRCLS) revisited: an analysis of 418 primarily managed cases. Ann Surg Oncol. 2012;19(4):1081-8.

30. De Cecco L, Negri T, Brich S, et al. Identification of a gene expression driven progression pathway in myxoid liposarcoma. Oncotarget. 2014; 5(15): 5965-77.

31. Nezu Y, Hagiwara K, Yamamoto $Y$, et al. miR-135b, a key regulator of malignancy, is linked to poor prognosis in human myxoid liposarcoma. Oncogene. 2016; 35(48): 6177-88.

32. Yu J, Sokumbi O. Cutaneous metastasis of pleomorphic liposarcoma to the scalp: an elusive diagnosis. J Cutan Pathol. 2016; 43(6): 526-30.

33. Wang L, Ren W, Zhou X, et al. Pleomorphic liposarcoma: a clinicopathological, immunohistochemical and molecular cytogenetic study of 32 additional cases. Pathol Int. 2013; 63(11): 523-31.

34. Mariño-Enríquez A, Hornick JL, Dal Cin P, et al. Dedifferentiated liposarcoma and pleomorphic liposarcoma: a comparative study of cytomorphology and MDM2/CDK4 expression on fine-needle aspiration. Cancer Cytopathol. 2014; 122(2): 128-37.

35. Ramírez-Bellver JL, López J, Macías E, et al. Primary dermal pleomorphic liposarcoma: utility of adipophilin and MDM2/CDK4 immunostainings. J Cutan Pathol. 2017; 44(3): 283-8

36. Ghadimi MP, Liu P, Peng T, et al. Pleomorphic liposarcoma: clinical observations and molecular variables. Cancer. 2011; 117(23): 5359-69.
37. Barretina J, Taylor BS, Banerji S, et al. Subtype-specific genomic alterations define new targets for soft-tissue sarcoma therapy. Nat Genet. 2010; 42(8): 715-21.

38. Lai JP, Rosenberg AZ, Miettinen MM, et al. NY-ESO-1 expression in sarcomas: a diagnostic marker and immunotherapy target. Oncoimmunology. 2014; 1(8): 1409-10.

39. Thway K, Flora R, Shah C, et al. Diagnostic utility of p16, CDK4, and MDM2 as

an immunohistochemical panel in distinguishingwell-differentiated and dedifferentiated liposarcomas from other adipocytic tumors. Am J Surg Pathol. 2012; 36(3): 462-9.

40. Kang Y, Horvai AE. p16 immunohistochemistry is less useful than MDM2 and CDK4 to distinguish dedifferentiated liposarcomas from other retroperitoneal mimics. Appl Immunohistochem Mol Morphol. 2017; 25(1): 58-63.

41. Kammerer-Jacquet SF, Thierry S, Cabillic F, et al. Differential diagnosis of atypical lipomatous tumor/well-differentiated liposarcoma and dedifferentiated liposarcoma: utility of p16 in combination with MDM2 and CDK4 immunohistochemistry. Hum Pathol. 2017; 59: 34-40.

42. Dickson MA, Tap WD, Keohan ML, et al. Phase II trial of the CDK4 inhibitor PD0332991 in patients with advanced CDK4-amplifi ed well-differentiated or dedifferentiated liposarcoma. J Clin Oncol. 2013; 31(16): 2024-8.

43. Igarashi K, Kawaguchi K, Kiyuna T, et al. Metabolic targeting with recombinant methioninase combined with palbociclib regresses a doxorubicin-resistant dedifferentiated liposarcoma. Biochem Biophys Res Commun. 2018; 506(4): 912-7.

44. Klein ME, Dickson MA, Antonescu C, et al. PDLIM7 and CDH18 regulate the turnover of MDM2 during CDK4/6 inhibitor therapy-induced senescence. Oncogene. 2018; 37(37): 5066-78.

45. Ray-Coquard I, Blay JY, Italiano A, et al. Effect of the MDM2 antagonist RG7112 on the P53 pathway in patients with MDM2-amplified, well-differentiated or dedifferentiated liposarcoma: an exploratory proof-of-mechanism study. Lancet Oncol. 2012; 13(11): 1133-40.

46. Meric-Bernstam F, Saleh MN, Infante JR, et al. Phase I trial of a novel stapled peptide ALRN-6924 disrupting MDMX- and MDM2-mediated inhibition of WT p53 in patients with solid tumors and lymphomas. J Clin Oncol. 2017; 35 (Suppl 15): 2505.

47. Bauer TM, Gounder MM, Weise AM, et al. A phase 1 study of MDM2 inhibitor DS-3032b in patients with well/de-differentiated liposarcoma (WD/DD LPS), solid tumors (ST) and lymphomas (L). J Clin Oncol. 2018;36 (Suppl 15):11514.

48. Sriraman A, Dickmanns A, Najafova Z, et al. CDK4 inhibition diminishes p53 activation by MDM2 antagonists. Cell Death Dis. 2018; 9(9): 918.

49. Hanes R, Munthe E, Grad I, et al. Preclinical evaluation of the pan-FGFR Inhibitor LY2874455 in FRS2-amplified liposarcoma. Cells. 2019; 8(2): 189.

50. Taylor BS, DeCarolis PL, Angeles CV, et al. Frequent alterations and epigenetic silencing of differentiation pathway genes in structurally rearranged liposarcomas. Cancer Discov. 2011;1(7):587-97.

51. Doyle LA, Tao D, Mariño-Enríquez A. STAT6 is amplified in a subset of dedifferentiated liposarcoma. Mod Pathol. 2014; 27(9): 1231-7.

52. Park HK, Kim M, Sung $M$, et al. Status of programmed death-ligand 1 expression in sarcomas. J Transl Med. 2018; 16(1): 303.

53. Tawbi HA, Burgess M, Bolejack V, et al. Pembrolizumab in advanced soft-tissue sarcoma and bone sarcoma (SARC028): a multicentre, two-cohort, single-arm, open-label, phase 2 trial. Lancet Oncol. 2017; 18(11): 1493-501.

54. Pollack SM, Jungbluth AA, Hoch BL, et al. NY-ESO-1 is a ubiquitous immunotherapeutic target antigen for patients with myxoid/round cell liposarcoma. Cancer. 2012; 118(18): 4564-70.

55. Pollack SM. The potential of the CMB305 vaccine regimen to target NY-ESO-1 and improve outcomes for synovial sarcoma and myxoid/round cell liposarcoma patients. Expert Rev Vaccines. 2017; 17(2): 107-14.

56. Hemminger JA, Ewart Toland A, Scharschmidt TJ, et al. The cancer-testis antigen NY-ESO-1 is highly expressed in myxoid and round cell subset of liposarcomas. Mod Pathol. 2013; 26(2): 282-8.

57. Dong $\mathrm{M}, \mathrm{Bi} \mathrm{J}$, Liu $\mathrm{X}$, et al. Significant partial response of metastatic intra-abdominal and pelvic round cell liposarcoma to a small-molecule VEGFR-2 tyrosine kinase inhibitor apatinib. Medicine. 2016; 95(31): e4368.

58. Yan P, Sun M, Sun Y, Liu C. Effective apatinib treatment of pleomorphic liposarcoma: A case report. Medicine. 2017; 96(33): e7771.

59. Bill KL, Casadei L, Prudner BC, et al. Liposarcoma: molecular targets and therapeutic implications. Cell Mol Life Sci. 2016; 73(19): 3711-8.

60. Ugras S, Brill E, Jacobsen A, et al. Small RNA sequencing and functional characterization reveals MicroRNA-143 tumor suppressor activity in liposarcoma. Cancer Res. 2011; 71(17): 5659-69. 
61. Yu PY, Lopez G, Braggio D, et al. miR-133a function in the pathogenesis of dedifferentiated liposarcoma. Cancer Cell Int. 2018; 18: 89.

62. Mazzu YZ, Hu Y, Shen $Y$, et al. miR-193b regulates tumorigenesis in liposarcoma cells via PDGFR, TGF $\beta$, and Wnt signaling. Sci Rep. 2019; 9(1): 3197.

63. Borjigin $\mathrm{N}, \mathrm{Ohno} \mathrm{S}, \mathrm{Wu} \mathrm{W}$, et al. TLS-CHOP represses miR-486 expression, inducing upregulation of a metastasis regulator PAI-1 in human myxoid liposarcoma. Biochem Biophys Res Commun. 2012; 427(2): 355-60.

64. Gits CM, van Kuijk PF, Jonkers MB, et al. MicroRNA expression profiles distinguish liposarcoma subtypes and implicate miR-145 and miR-451 as tumor suppressors. Int J Cancer. 2014; 135(2): 348-61.

65. Zhang P, Bill K, Liu J, et al. MiR-155 is a liposarcoma oncogene that targets casein kinase-1a and enhances $\beta$-catenin signaling. Cancer Res. 2012; 72(7): 1751-62.

66. Vincenzi B, Iuliani M, Zoccoli A, et al. Deregulation of dicer and mir-155 expression in liposarcoma. Oncotarget. 2015; 6(12): 10586-91.

67. Kapodistrias N, Mavridis K, Batistatou A, et al. Assessing the clinical value of microRNAs in formalin-fixed paraffin-embedded liposarcoma tissues: overexpressed miR-155 is an indicator of poor prognosis. Oncotarget. 2017; 8(4): 6896-913.

68. Lee DH, Amanat S, Goff C, et al. Overexpression of miR-26a-2 in human liposarcoma is correlated with poor patient survival. Oncogenesis. 2013; 2: e47.

69. Casadei L, Calore F, Creighton CJ, et al. Exosome-derived miR-25-3p and miR-92a-3p stimulate liposarcoma progression. Cancer Res. 2017; 77(14): 3846-56. 